\section{Hartmann's Procedure And Its Outcome - An Analysis of 78 Consecutive Cases}

\section{ORIGINAL}

\section{Abstract}

Background: To analyse the outcome of patients who had Hartmanns procedure (HP) performed in a single institution.

Methods and findings: We retrospectively analysed 78 consecutive patients who had HP performed in our Hospital from $1^{\text {st }}$ January 2010 to $31^{\text {st }}$ December 2013 over a four year period. We analysed age, sex, indications, emergency or elective stoma formation, complications, reversal rate and complications of reversal. Mean age of these 78 patients with HP was 69 years (Range 34-92 years). Thirty five patients were females and 43 were males. In 61 (76.2\%) patients HP was done as an emergency and in $17(23.8 \%)$ it was done on an elective basis. Out of these 78 patients, 40 (47.2\%) had complicated diverticular disease and 25 (34.7\%) had locally advanced or obstructed rectosigmoid carcinoma. Out of 78 patients who had HP, 12 died in the postoperative period. Nine (12.5\%) patients developed parastomal hernia. One each had ischaemia, prolapse and stenosis. Only $18(23.07 \%)$ patients had their Hartmann's reversal (HR) done, all of these were by open method. Commonest complication following Hartmann's reversal was hernia at the stoma site which was seen in three patients.

Conclusion: HP has a high mortality due to the primary disease process. It has a high rate of long term complications like parastomal hernia. In our series only $23.07 \%$ patients had their Hartmann's reversed.

\author{
Sanjay Dalmia ${ }^{1}$ \\ 1 Department of Surgery, Stafford Hospital. \\ Weston Road, Stafford, ST.
}

\section{Contact information:}

Sanjay Dalmia.

झdalmiasanjay@hotmail.com

\section{Keywords}

Hartmann's, outcome, reversal, complications

\title{
Introduction
}

$\mathrm{HP}$ is a common operation in management of colorectal emergencies particularly in left colonic pathologies. It is also performed electively 
in selected cases. HP is relatively easy to perform and is used for prevention of an intestinal anastomosis in patients with intra-abdominal sepsis. From patient's point of view it is a major responsibility and a change in their social, working and religious lives.

\section{Methods}

We retrospectively reviewed 78 consecutive patients with HP that were done in the period from 01/01/2010 to 31/12/2013. We included all patients who had either emergency or elective HP performed in this period. We noted their age, sex, indication for which they had HP done. We recorded number of patients who developed complications like ischaemia, stenosis, prolapse and parastomal hernia. We also recorded those patients who had HP performed, whether they were subsequently reversed or not. We recorded complications of Hartmann's reversal (HR) like leakage, intestinal obstruction, wound infection and incisional hernia at stoma site in patients who had HR done.

\section{Results}

Of the 78 patients who had HP done, 15 patients died within 30 days of their surgery from their presenting illness. All of these patients presented with acute intra abdominal sepsis. Mean age of 78 patients with HP was 69 years (Range 34-92 years). Thirty five $(44.8 \%)$ of these 78 patients were females and 43(55.2\%) were males. 61(76.2\%) were done as an emergency and 17(23.85\%) were done on an elective basis. Out of the total 78 patients, $40(51.2 \%)$ had complicated diverticular disease and 25 (32.05\%) had locally advanced or obstructed recto sigmoid carcinoma. Three (3.8\%) patients each had ovarian carcinoma and colovesical fistula. Two patients each had colovaginal fistula, and stercoral perforation. One patient each had sigmoid volvulus, strangulated left inguinal hernia and splenic flexure carcinoma. (Table-1)
Table 1. Indications of Hartmann's Procedure.

\begin{tabular}{|l|l|}
\hline \multicolumn{1}{|c|}{ Indications } & No. of cases \\
\hline Diverticular disease & 40 \\
\hline Recto sigmoid Carcinoma & 25 \\
\hline Ovarian Carcinoma & 03 \\
Colovesical fistula & 03 \\
\hline Colovaginal fistula & 02 \\
\hline Stercoral Perforation & 02 \\
\hline Strangulated left inguinal hernia & 01 \\
Sigmoid Volvulus & 01 \\
\hline Splenic flexure Carcinoma & 01 \\
\hline
\end{tabular}

Nine $(12.5 \%)$ patients developed parastomal hernia. One each had ischaemia, prolapse and stenosis. (Table-2)

Table 2. Complications of Hartmann's Procedure.

\begin{tabular}{|c|c|}
\hline Indications & No. of cases \\
\hline Parastomal Hernia & 09 \\
\hline Ischaemia & 01 \\
\hline Prolapse & 01 \\
\hline Stenosis & 01 \\
\hline
\end{tabular}

Average time for Hartmann's reversal (HR) was 352 days with a range of 105 to 2190 days. Out of 63 patients who were discharged from hospital after HP, 18 were subsequently reversed. Most common reason for non-reversal of stoma was one or more co morbid factors like stroke, myocardial infarction and angina in sixteen (20.5\%) patients. Nine (11.5\%) patients were considered fit for HR but they were happy with their stoma and did not want to have reversal. Only eighteen (23.07\%) out of 78 patients had their Hartmann's reversed. Remaining twenty patients, who did not have reversal, cause for non- reversal was not clear from their notes. Most common complication following Hartmann's reversal was hernia at the stoma site in four patients. One patient developed minor postoperative leak that settled conservatively and one had colovesical fistula after HR. 


\section{Discussion}

$\mathrm{HP}$ is a good option for surgical treatment in complicated recto sigmoid pathologies, particularly diverticular disease or obstructed cancers. The morbidity and mortality of this operation is highly dependent on the degree of preoperative sepsis and the patient's preexisting condition [1]. Complications after HP are common and can be early complications like ischaemia or late complications like stenosis, prolapse and parastomal hernia. Up to 30\% of patients with a stoma have been shown to need surgical re intervention in first 10 years [2]. Studies have shown that $88 \%$ of patients with diverticular disease had primary anastomosis after resection of sigmoid colon though $86 \%$ in this series were done electively. [3]. Some studies have quoted a HR rate of $46 \%$ though in our study only $18(23.07 \%)$ patients had reversal out of 78. [4]. Another retrospective study, very similar to ours, showed that $63 \%$ of HP was done as an emergency and $37 \%$ as elective procedures. In our study this percentage was $76.2 \%$ and $23.8 \%$ respectively. The overall postoperative mortality in this study was $10 \%$, with no significant difference between the emergency and the elective group. Patients who were suitable for reversal, only $70 \%$ had it done with no postoperative death or anastomotic leakage after reversal. [5] This multi centre study showed that $35.2 \%$ patients who had undergone reversal after HP were younger and had a low American Society of Anaesthetists (ASA) score. Complications occurred in $45.2 \%$ of these patients and were associated with age, diabetes mellitus, arteriosclerosis, obesity, smoking, chemotherapy and COPD. [6] This study attempted to quantify the risk of non-closure by deriving a predictive scoring system for Hartmann's reversal and patients were separated into those who underwent Hartmann's reversal within one year and those who did not. Age, ASA score, pulmonary co morbidity, preoperative blood transfusion, perforation, and anticoagulants were factors for non-reversal. All patients with a predictive score of less than 14 had their
Hartmann's reversed and $88 \%$ with scores greater than 18 did not have it reversed. [7] $72.5 \%$ of emergency Hartmann's were performed for benign diseases whereas $68.9 \%$ of the elective Hartmann's were performed for cancer in this series. $23.3 \%$ of these patients underwent reversal with a median time interval of 284.5 days. [8] In our series time of reversal of Hartmann's varied from 105 days to 2190 days with a mean of 352 days. Emergency HP showed that mortality and morbidity rates were $19 \%$ and $65 \%$, respectively. Age and respiratory disease affected mortality rates whilst hypertension and cardiac disease affected morbidity rates. 40\% of these patients had reversal after a median of 9 months. $(p=0.011)$. [9] Patients $>60$ years old who had HR had significantly longer length of hospital stay compared to rest of the group. There were no differences in outcomes between groups based on ASA score or the presence of multiple preoperative comorbidities. An albumin level of $<3.5$ was the only significant predictor of postoperative complications. [10] Of all the patients who underwent HP, reversal was performed in $25.9 \%$ with a mean time of 13.3 months. There were no deaths but $54.8 \%$ suffered complications. Male sex, non-neoplastic disorder, younger age and lower anaesthetic risk were factors favouring a reversal. [11]

Retrospective analysis from a single centre assessing the outcome of HR showed that patients who underwent reversal were mostly ASA 2 (60\%), with very few being ASA 4 (4.6\%). There was a significant influence of hypertension, smoking and ASA grade on complications after HR. [12] A single-center study of a prospective colorectal surgery database showed that in the open HR group mean operative time, the estimated blood loss and complication rate at one and twelve months were significantly higher compared to laparoscopic HR group. Open HR also had $56 \%$ higher costs compared to the laparoscopic HR, when combining the surgery-related costs and the length of hospital stay.[13] A systematic review with eight comparative studies showed that laparoscopic 
HR has a significantly reduced complication rate ( $P$ $<0.01)$, intra-operative blood loss $(P<0.001)$ and hospital stay $(P<0.01)$ compared with open HR. [14] Hartmann's procedure for rectal cancer was done in patients who were significantly older, had higher ASA-score, poorer WHO performance score and lower serum albumin levels. Operative time for HP was shorter than after anterior resection and had less bleeding. Few patients developed pelvic complications despite a higher age, more co-morbidities, metastases and functional compromise when compared with the patient who had anterior resection or abdominoperineal excision. HP is a useful alternative procedure in the old and frail patients with rectal cancer. [15]

While HP is the most commonly performed operation for acute diverticulitis, primary anastomosis with (PAPD) or without (PA) proximal diversion are also performed in appropriate patients. Thirty-day mortality was $7.3 \%, 1.6 \%$ and $4.6 \%$ for HP, PAPD, and PA respectively while surgical site infections occurred in $19.7 \%, 13.2 \%$ and $17.9 \%$ respectively in these patients. After adjusting for age, alcohol consumption, comorbidities, steroid use, preoperative laboratory values, hemorrhage at admission and laparoscopic surgery three groups did not have significantly different risk of infective surgical complications, acute kidney injury, cardiovascular incidents, or venous thrombo embolism. [16] A systematic review containing 14 studies was conducted to compare different surgical treatments for Hinchey III or IV diverticulitis. Primary resection with anastomosis had a significant advantage in terms of lower mortality rate with respect to HP and a shorter length of stay. Morbidity and hospital stay were further lower in the laparoscopic peritoneal lavage group compared to the primary resection and anastomosis group. [17] Patients with laparoscopic HP had significantly longer hospital stay compared to LLD ( $P$ $<0.01)$. Though both laparoscopic HP and LLD can be performed safely and effectively for managing severe diverticulitis with generalized peritonitis, LLD showed significantly better short- and long-term clinical outcomes for managing perforated diverticulitis. [18] Hartmann's procedure for perforated diverticulitis is associated with significant morbidity and mortality. Independent predictors of morbidity were Hinchey IV $(P<0.001)$ and hypo proteinaemia $(P=0.001)$ and for mortality were ASA $>3$, abnormal creatinine, steroid use, Hinchey IV, low albumin and low body mass index. Efforts should focus on the identification of patient benefiting from early elective surgery and alternative surgical approaches in perforated diverticulitis. [19] Anastomotic leakage after PA was associated with patient co-morbidity rather than with Hinchey category, suggesting that the decision to perform PA should be guided by patient's general condition rather than on the extent of intra abdominal inflammation. [20] Primary resection and anastomosis with or without proximal diversion and laparoscopic lavage are alternatives to HP. Change in surgical approach needs large multi centered prospective randomized trial to determine the best strategy in emergency management of diverticulitis. [21]

\section{Conclusion}

HP has an invaluable role in appropriate cases but has a considerable morbidity and mortality in these patients who are very unwell. They should be reversed where suitable to improve body image and quality of life. The morbidity of stoma closure is appreciable with hernia at stoma site being most common. In a large no. of patients HP is never reversed even though the primary disease is under control.

\section{Conflict of interest}

None

\section{Acknowledgement}

None 


\section{References}

1. Gonzalez OA, Duran RO, Avalos GJ, Herrara CG, Orozoo MA, Gutierrez DR, et al. Hartmann's Procedure: Institutional experience with 92 consecutive cases. Rev-Gastroenterol-Mex 1999; 64(3): 127-133.

2. Kuijpers JH. Gastrointestinal surgery and gastroenterology. xi. Stoma and stoma surgery. Nederlands tijdschrift voor geneeskunde 2001; 145(24): 1144-8.

3. Belmonte C, Klas JV, Perez JJ, Wong WD, Rothenberger DA, Goldberg SM, et al. Hartmann's Procedure-. First choice or last resort in diverticular disease? Arch-Surg.1996; 131(6): 612-615.

4. Bisogno ML, Puntillo G, Andrich R, Grifoni G, Rabuffi F, Ruotolo F. Colorectal reconversion after Hartmann's Procedure. Apropos of 26 cases Ann-Ital-Chir.1998; 69 (3): 341-5.

5. Khosraviani K, Campbell WJ, Parks TG, Irwin ST. Hartmann procedure revisited. The European journal of surgery.2000; 166 (11): 878-81.

6. Roig JV, Cantos M, Balciscueta Z, Uribe N, Espinosa J, Roselló $V$, et al. Sociedad Valenciana de Cirugía Cooperative Group. Hartmann's operation: how often is it reversed and at what cost? A multicentre study. Colorectal Dis. 2011; 13(12): 396402.

7. Riansuwan W, Hull TL, Millan MM, Hammel JP. Non reversal of HP for diverticulitis: derivation of a scoring system to predict non reversal. Dis Colon Rectum. 2009; 52(8): 1400-8

8. David GG, Al-Sarira AA, Willmott S, Cade D, Corless DJ, Slavin JP. Use of HP in England. Colorectal Dis. 2009; 11(3): 308-12.

9. Leong QM, Koh DC, Ho CK. Emergency Hartmann's procedure: morbidity, mortality and reversal rates among Asians. Tech Coloproctol. 2008; 12(1): 21-5.

10. Schmelzer TM, Mostafa G, Norton HJ, Newcomb WL, Hope WW, Lincourt $A E$, et al. Reversal of Hartmann's procedure: a high-risk operation? Surgery.2007; 142(4): 598-606.

11. Roque CC, Marchena GJ, Hemmersbach MM, Acosta MA, Rodriguez MA, Fariña $C R$, et al. Analysis of the factors related to the decision of restoring intestinal continuity after Hartmann's procedure. Int J Colorectal Dis. 2007; 22(9): 1091-6.

12. Banerjee S, Leather AJ, Rennie JA, Samano N, Gonzalez JG, Papagrigoriadis S. Feasibility and morbidity of reversal of Hartmann's. Colorectal Dis. 2005; 7(5): 454-9.

13. De'angelis N, Brunetti F, Memeo R, Batista da Costa J, Schneck AS, Carra MC, et al. Comparison between open and laparoscopic reversal of HP for Diverticulitis. World J Gastrointest Surg. 2013; 5(8): 245-51.

14. Siddiqui MR, Sajid MS, Baig MK. Open vs laparoscopic approach for reversal of Hartmann's procedure: a systematic review. Colorectal Dis. 2010; 12(8): 733-41.

15. Sverrisson I, Nikberg M, Chabok A, Smedh K. Hartmann's procedure in rectal cancer: a population-based study of postoperative complications. Int J Colorectal Dis. 2015; 30(2): 181-6.
16. Tadlock MD, Karamanos E, Skiada D, Inaba K, Talving $P$, Senagore $A$, et al. Emergency surgery for acute diverticulitis: which operation? A National Surgical Quality Improvement Program study. J Trauma Acute Care Surg. 2013; 74(6): 1385-91.

17. Cirocchi R, Trastulli S, Desiderio J, Listorti C, Boselli C, Parisi A, et al. Treatment of Hinchey stage III-IV diverticulitis: a systematic review and meta-analysis. Int J Colorectal Dis. 2013;28(4): 447-57.

18. Liang S, Russek K, Franklin ME Jr. Damage control strategy for the management of perforated diverticulitis with generalized peritonitis: laparoscopic lavage and drainage vs. laparoscopic Hartmann's procedure. Surg Endosc. 2012; 26(10): 2835-42.

19. Ince M, Stocchi L, Khomvilai S, Kwon DS, Hammel JP, Kiran RP. Morbidity and mortality of the Hartmann procedure for diverticular disease over 18 years in a single institution. Colorectal Dis.2012; 14(8): 492-8.

20. Mueller MH, Karpitschka M, Renz B, Kleespies A, Kasparek MS, Jauch $\mathrm{KW}$, et al. Co-morbidity and postsurgical outcome in patients with perforated sigmoid diverticulitis. . Int J Colorectal Dis. 2011; 26(2): 227-34.

21. Bauer VP. Emergency management of diverticulitis Clin Colon Rectal Surg. 2009; 22(3): 161-8

\section{Comment on this article:}

\section{(f) $[$ in $8+\mathbf{S} P$}

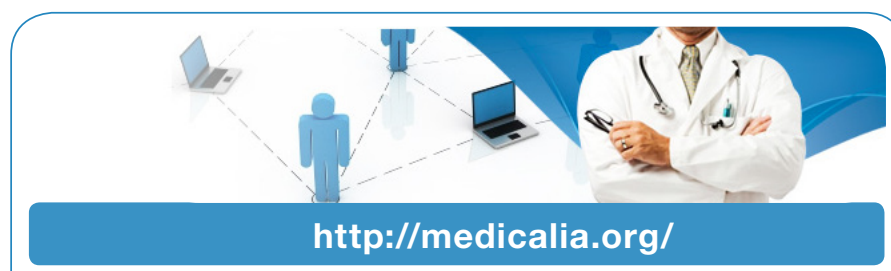

Where Doctors exchange clinical experiences, review their cases and share clinical knowledge. You can also access lots of medical publications for free. Join Now!

\section{Publish with iMedPub}

http://www.imed.pub

International Archives of Medicine is an open access journal publishing articles encompassing all aspects of medical science and clinical practice. IAM is considered a megajournal with independent sections on all areas of medicine. IAM is a really international journal with authors and board members from all around the world. The journal is widely indexed and classified Q1 in category Medicine. 